\title{
Single Center Study of Vaccination Breakthrough Infection with SARS-CoV-2 Among Erbil Population in August 2021
}

\author{
Zakarea Abdullah Yaseen Al-khayat*, Sharmeen Qadr Faqi², Dlshad Abdullah Hasan², Dalia Yaqoub Falo²
}

'Department of Microbiology, College of Medicine, Hawler Medical University, Erbil, Iraq

2Molecular Diagnostic Department, Central Public Health Laboratory (CPHL), Erbil, Iraq.

*Correspondence to: Zakarea Abdullah Yaseen Al-khayat (E-mail: zakarea.abdullah@hmu.edu.krd)

(Submitted:05 September 2021 - Revised version received: 18 September 2021 - Accepted: 09 October 2021 - Published online: 26 October 2021)

\begin{abstract}
Objectives: This is a first study to determine the incidence of COVID-19 infection in the post-vaccinated in Erbil City population.

Methods: This prospective study was conducted in the Central Health Laboratory in Erbil City over a period from 1st to 31 st of August 2021.

All vaccinated \& non vaccinated persons (18 years of age and above) who had symptoms suggestive of COVID-19 were engaged in the study. Nasopharyngeal swabs were collected and examined for SARS-CoV-2 by real-time RT-PCR implemented to all attendance according to World Health Organization guidelines.

Results: A total of 2934 persons had attended the Central Laboratory for checking during the study period. From this total number, 551 (18.8\%) were vaccinated while 2383 were nonvaccinated (81.2\%). Sixty one persons $(61,11.1 \%)$ of the vaccinated group showed a positive PCR. The highest incidence of PCR positivity according to age range, gender and vaccine dose, were as follows: male (42; 68.9\%), 33-47 years $(36 ; 59 \%)$, second dose $(36 ; 59 \%)$ respectively. Statistically, the differences of distribution of the PCR positivity concerning the these factors were: non-significant. The higher incidence of PCR positivity was among the nonvaccinated group (449, 18.8\%) and statistically, the differences of distribution of the PCR positivity between both groups of vaccinated and the nonvaccinated was significant $(P \leq 0.05)$.

Conclusion: Primarily the present study conclude that SARS-CoV-2 incidence among vaccinated persons was $11.1 \%$. Overall the present data provides further assurances of the effectiveness of the vaccines even when the vaccine was not able to prevent completely the infection. Further studies are needed to explore this topic.

Keywords: SARS-CoV-2, vaccines, polymerase chain reaction, post-vaccination, breakthrough, Erbil
\end{abstract}

\section{Introduction}

COVID-19 is a novel infectious malady caused by a SARS-CoV-2 virus, which is demonstrated mostly as an acute respiratory disease with an interstitial alveolar pneumonia. Yet, it can involve different organs encompassing the heart, kidneys, nervous system, blood and the digestive tract $^{1}$.

COVID-19 vaccines are efficient and pivotal weapons to boost the control of this pandemic ${ }^{2}$. Vaccination against COVID-19 has been considered as the most influential mean to halt the dispersal of the virus, beside the protective measures utilized by each individual ${ }^{3}$.

The COVID-19 mRNA vaccines (from Biontech/Pfizer and Moderna) have been authorized in different countries (including the USA and the EU) after successful clinical trials and are already in prevalent use $\mathrm{e}^{4}$. The two products contain a nucleoside-modified mRNA, encoding the sequence of the full-length $S$ protein with two stabilizing proline mutations in S2 to preserve the native prefusion conformation. Both vaccines use lipid nanoparticles for delivery ${ }^{5}$. In the real-world settings, the Pfizer-BNT162b2 vaccine is approximately $90 \%$ effective in preventing SARS-CoV-2 infection and $94-100 \%$ effective in preventing severe or fatal disease ${ }^{6}$.

Meanwhile, several vaccines based on different nonreplicating adenovirus vectors and the full-length $S$ protein are used for vaccination campaigns in many countries after approval by national and international authorities ${ }^{3}$. These vaccines include products of the Gamaleya Institute in Moscow (Sputnik V), University of Oxford/AstraZeneca (AstraZeneca, Cambridge, UK) (ChAdOx1-S/AZD1222), the Beijing Institute of Biotechnology/CanSino (CanSino Biologics, Tianjin, China) and Janssen Pharmaceutica (Janssen Pharmaceutica,
Beerse, Belgium $)^{7}$. In these vaccines different adenoviruses are used as vectors but the basic principle of production platforms and mechanism of action is the same ${ }^{8}$. The gene for the SARS-CoV-2 S protein is synthesized as a DNA and engineered into the DNA genome of adenoviruses, replacing an adenovirus gene (E1) that is essential for virus replication? Through this manipulation, the adenovirus can no longer replicate and cannot give rise to a full infectious cycle (it is therefore referred to as non-replicating viral vector), but it can still enter cells and express the inserted foreign gene to produce the coronavirus S protein ${ }^{10,11}$.

However, early studies propose that COVID-19 vaccines shelter against severe illness; however, postvaccination SARS-CoV-2 infections (i.e., breakthrough infections) can occur because COVID-19 vaccines do not offer 100\% protection $^{12}$. More recently, postvaccination infections in fully vaccinated persons have been mentioned though little is known about the risk factors, clinical presentation and outcomes for breakthrough infections compared with demographically and clinically similar controls ${ }^{13}$. Thus, the objectives of this study were to report any postvaccination infections among fully or partially vaccinated persons, and to determine some factors like: age, gender, and vaccine dose which may have an association with infections in the vaccinated persons. Our aim was not to determine specific vaccine efficacy or effectiveness.

\section{Materials and Methods}

\section{Study Protocol}

This prospective study was carried out in the Molecular diagnostic department at the Central Public Health Laboratory 
(CPHL) in Erbil City through a period from August 12021 to August 31 2021. This study was achieved with the cooperation of Department of Microbiology, College of Medicine, Hawler Medical University, Erbil, Iraq.

\section{Moral Considerations}

This study was confirmed by the: Ethics Committee of Hawler Medical University, Erbil and approved by Erbil director health for collecting samples and data from referred patients at CPHL-Erbil. Acquainted endorsement was possessed from each patient. The patients were aware of study's goals and they could regress thereof if they wished so to do.

\section{Study Population}

The study population included all suspected cases of Covid-19 attending CPHL for PCR screening of SARS-CoV 2 infection during study period. Inclusion criteria were: 18 year old and above, and inhabitants of Erbil governorate. All the attendance who conferred agreement for SARS-2 PCR testing and to share in this study were involved in the study. Relevant clinical information, including patients' vaccination status (type and dose number) age, gender, date of birth, was collected upon recruitment. Non-consenting or unwilling patients were excluded from this study.

\section{SARS-CoV-2 Testing by Real-time RT-PCR}

Nasopharyngeal and/or throat swab samples were collected in viral transport medium from the enrolled patients by a trained laboratory technician and examined for SARS-CoV-2 by real-time RT-PCR following World Health Organization guidelines.

Viral RNA was extracted using SphaeraMag DNA/RNA Isolation Kit on automate Phoenix-Pure96 system (procomcure com.) following manufacture's instruction. Real-time RT-PCR was done using DIAGNOVITAL ${ }^{\circ}$ SARS-CoV-2 RealTime PCR Kit (RTA Laboratories Biological Products Pharmaceutical and Machinery Industry) on Rotor-Gene $Q$ (QIAGEN) Real-Time PCR Detection System. DIAGNOVITAL ${ }^{\circ} A R S-C o V-2$ detects the presence of 2 different and highly specific gene sequences of SARS-CoV-2: E gene and RdRp gene. All 2 assays must be tested positive to confirm the sample as SARS-CoV-2-positive.

\section{Statistical Analysis}

The data analysis was performed using descriptive statistics, including frequency, and frequency percentage. Comparisons were made using chi 2 test by using standard equations. The results were announced with $P \leq 0.05$ or $P \leq 0.01$ as the acceptable level of significance.

\section{Results}

A total of 2934 persons had attended the Central Laboratory for checking during the study period. Of this total number, $551(18.8 \%)$ were vaccinated while 2383 were nonvaccinated (81.2\%).

Table 1 delineates the incidence of PCR positivity among vaccinated persons according to age, gender and number of dose. It is noted that sixty one persons (11.1\%) of the vaccinated group showed a positive PCR. The highest incidence of PCR positivity according to age range, gender and dose, were as follows: male $(42 ; 68.9 \%), 33-47$ years $(36 ; 59 \%)$, second

\begin{tabular}{|c|c|c|c|c|c|}
\hline Parameter & $\mathrm{N}$ & PCR (+) & $\%$ & PCR (-) & $\%$ \\
\hline \multicolumn{6}{|l|}{ Age } \\
\hline $18-32$ & 171 & 18 & 29.5 & 153 & 31.2 \\
\hline $33-47$ & 301 & 36 & 59 & 265 & 54.1 \\
\hline $48-62$ & 54 & 3 & 4.9 & 51 & 10.4 \\
\hline $63-82$ & 25 & 4 & 6.6 & 21 & 4.3 \\
\hline \multirow[t]{2}{*}{ Total } & 551 & 61 & 100 & 490 & 100 \\
\hline & & $d f=3 N S$ & & & \\
\hline \multicolumn{6}{|l|}{ Gender } \\
\hline Male & 421 & 42 & 68.9 & 379 & 77.3 \\
\hline Female & 130 & 19 & 31.4 & 111 & 22.7 \\
\hline \multirow[t]{2}{*}{ Total } & 551 & 61 & 100 & 490 & 100 \\
\hline & & $\mathrm{df}=1 \mathrm{NS}$ & & & \\
\hline \multicolumn{6}{|l|}{ Dose } \\
\hline First & 179 & 25 & 41 & 154 & 31.4 \\
\hline Second & 372 & 36 & 59 & 336 & 68.6 \\
\hline \multirow[t]{2}{*}{ Total } & 551 & 61 & 100 & 490 & 100 \\
\hline & & $\mathrm{df}=1 \mathrm{NS}$ & & & \\
\hline
\end{tabular}

\begin{tabular}{|c|c|c|c|c|c|}
\hline Parameter & Vaccinated & $\%$ & Nonvaccinated & $\%$ & Total \\
\hline $\operatorname{PCR}(+)$ & 61 & 11.1 & 449 & 18.8 & 510 \\
\hline PCR (-) & 490 & 88.9 & 1934 & 81.2 & 2424 \\
\hline Total & 551 & 100 & 2383 & 100 & 2934 \\
\hline
\end{tabular}

$X^{2}=18.8187 \mathrm{df}=1$ Significant $(P \leq 0.05)$

dose $(36 ; 59 \%)$ respectively. Statistically, the differences of distribution of the PCR positivity concerning the abovementioned factors were: non-significant.

Table 2 clarified the incidence of PCR positivity among vaccinated persons in comparison to the nonvaccinated persons (control). The higher incidence of PCR positivity was among the nonvaccinated group $(449,18.8 \%)$ and statistically, the differences of distribution of the PCR positivity between both groups of vaccinated and the nonvaccinated was significant $(P \leq 0.05)$.

\section{Discussion}

To the best of our knowledge, no published data are available on the occurrence of SARS-CoV-2 infections among vaccinated citizens in Erbil governorate, hence this study can be considered as the first study of such quality to deal with and investigate the incidence, correlated factors among these persons.

The incidence of positive PCR in the post-vaccinated group was $11.1 \%$, which was differed significantly from the incidence in the nonvaccinated group (18.8\%). It seems that from the results of the present study, factors like age, gender and dose had no impact on the high incidence of PCR positivity among vaccinated group. 
Post-vaccinations infections are a matter of interest but sufficient data concerning these infections are not available in real world setting ${ }^{14}$. Vaccines have effectiveness in decreasing risk of getting COVID-19 infections by $70-90 \%$, and also shield from severe infection ${ }^{13}$. It is possible, therefore, some people who are fully vaccinated against COVID-19 may get COVID-19 infection ${ }^{4}$.

A recent study by Amit et al. ${ }^{15}$ showed that the ratio of vaccinated people infected with COVID-19 after receiving the first dose vaccine was $0.5 \%$, while a study achieved by Hatmal $\mathrm{MM}$ et al. in Jordan ${ }^{16}$ declared that the incidence was $7.02 \%$ after the first dose only. This variation in ratios could be due to the fact that Amit et al.s study involved only healthcare workers; this class of the population is assumed to be welleducated and has a furthermore strict commitment in following prevention regulations to avoid COVID-19 infection. In Amit's study, individuals infected with COVID-19 were also tested in the early post-vaccination period (1-10 days), while there was no time limit in the present study.

Anecdotal report (unpublished) from India \& declared records from other part of world (refer website of Center for Disease Control, USA) deduce that these infections are existing. In addition, it seems that these breakthrough infections are either asymptomatic or mild in nature ${ }^{14}$.

Despite the fact that the preventive efficacy of COVID-19 vaccines is argued in clinical trials, the knowledge about what happens following vaccination in the real world is still modest, especially among the general population ${ }^{16}$. Thus, knowing what to expect after vaccination will help with public education, dispelling myths, and lowering the apprehension about COVID-19 vaccines ${ }^{15}$. Fear and suspicion, as well as a lack of information about clinical trials, have all been identified as factors that may lead to hesitancy in receiving the COVID-19 vaccines $^{10}$.

The present study found a relatively high SARS-CoV-2 following receipt of the second dose. Polack et al., ${ }^{4}$ and Butt AA et al. ${ }^{13}$ concluded that while the vaccine's protective effect may not be obvious during the first two weeks after vaccination so recipients may wrongly realize themselves to be at a reduced risk of SARSCoV-2 infection and become less firm to nonpharmacological preventive measures such as social distancing and face covering.

Careful education and counselling during the vaccination process could help efforts to minimize such risk-compensation behavior ${ }^{17}$.

The concept of vaccination is dependent on triggering an adaptive immune response, $\mathrm{B}$ and $\mathrm{T}$ cells, from which memory cells will evolve and furnish a long-lasting immunity. Although an immune response can take place as early as within the first week, long-lasting immunity can take up to 4 weeks to develop ${ }^{7}$. The BNT162b2 vaccine was shown to elicit an effective humoral (antibody-mediated) and cellular (T-cell-mediated) responses a week after the booster dose. However, the response between the first and second doses was negligible ${ }^{18}$.

Vaccine efficacy does not always portend vaccine effectiveness, i.e., the protection advantage related to a vaccine when administered non-randomly under field conditions, Equally, randomized controlled trials were done in a nominated age group, or geographical setting might not predict effectiveness if the vaccine is more widely prevailed. Alternative vaccine platforms or the addition of adjuvants may be required for adequate immunogenicity, as for influenza vaccines ${ }^{19,20}$

It is noted from the results of the present studies, old ages persons had a lower incidence of PCR positivity while the highest incidence was in the age range of 33-47 years (59\%). A study by Butt AA et al. ${ }^{21}$ in Qatar announced that increasing age was independently associated with a higher risk of severe disease or death in persons with breakthrough infection. In addition, encumbrance of comorbidities was not linked with the higher risk of severe disease or death ${ }^{21,22}$. It is worthy to mention that Central Laboratory in Erbil offers its services for public and military personals so it is expected that most attendance will be males and of age ranges mostly detected in the present study.

The strengths of our study include: a national population, appropriately matched control group, it is the first step to elucidate such an important and newly arising topic in this vaccination campaign.

Limitations of the present study include small sample size, short duration, bias and shortages in collecting data regarding demography and risk factors like on obesity, smoking and co-morbid diseases which are important determinants of severity of COVID-19.

Further studies are recommended to study and investigate this vital topic taking into consideration type of the vaccine, interval between the emergence of symptoms and the last dose. In addition follow up such persons are mandatory to explore any complications associated with vaccination.

\section{Acknowledgments}

The authors are grateful to the management of Health Directorate of Erbil for their allowing us to conduct the study. A words of thank are directed to the staff of the Central Laboratory for their assistance. Our thanks and regards to the people who share in the study and we appreciate their patience during the study.

\section{Financial Support and Sponsorship}

Nil.

\section{Conflicts of Interest}

There are no conflicts of interest.

\section{References}

1. Dawood H, Hwayyiz A, Ibrahim I, Rahman IA. The clinical features of COVID-19 in a group of Iraqi patients: A record review. Journal of the Faculty of Medicine Baghdad. 2021 May 11;63(1):8-12.

2. Baden LR, El Sahly HM, Essink B, et al. Efficacy and Safety of the mRNA-1273 SARS-CoV-2 Vaccine. N Engl J Med 2021; 384(5):403-16.

3. Voysey M, Clemens SAC, Madhi SA, et al. Safety and efficacy of the ChAdOx $n C O V-19$ vaccine (AZD1222) against SARS-CoV-2: an interim analysis of four

randomised controlled trials in Brazil, South Africa, and the UK. The Lancet 2021;397(10269): 99-111.

4. Polack FP, Thomas SJ, Kitchin N, Absalon J, Gurtman A, LockhartS, et al. Safety and efficacy of the BNT162b2mRNA Covid-19 vaccine. N Engl J Med. 2020;383(27):2603-15.

5. Dagan N, Barda N, Kepten E, et al. BNT162b2 mRNA Covid-19 Vaccine in a Nationwide Mass Vaccination Setting. N Engl J Med 2021;384:1412-23. 
6. Hacisuleyman E, Hale C, Saito Y, et al. Vaccine Breakthrough Infections with SARS-CoV-2 Variants. N Engl J Med 2021;384:2212-18.

7. Heinz FX, Stiasny K. Profiles of current COVID-19 vaccines. Wiener Klinische Wochenschrift. 2021 Apr;133(7):271-83.

8. Zhu F-C, Guan X-H, LiY-H, Huang J-Y, Jiang T, Hou L-H, et al. Immunogenicity and safety of a recombinant adenovirus type-5-vectored COVID-19 vaccine in healthy adults aged 18 years or older: a randomised, double-blind, placebo controlled, phase 2 trial. Lancet. 2020;396(10249): 479-88.

9. Logunov DY, Dolzhikova IV, Shcheblyakov DV, Tukhvatulin Al, Zubkova OV, Dzharullaeva AS, Kovyrshina AV, Lubenets NL, Grousova DM, Erokhova AS, Botikov AG. Safety and efficacy of an rAd26 and rAd5 vector-based heterologous prime-boost COVID-19 vaccine: an interim analysis of a randomised controlled phase 3 trial in Russia. The Lancet. 2021 Feb 20;397(10275):671-81.

10. Voysey M, Clemens SAC, Madhi SA, Weckx LY, Folegatti PM, Aley PK, et al. Safety and efficacy of the ChAdOx1 nCoV- 19 vaccine (AZD1222) against SARS-CoV-2: an interim analysis of four randomised controlled trials in Brazil, South Africa, and the UK. Lancet. 2021;397(10269):99-111.

11. Sadoff J, Le Gars M, Shukarev G, Heerwegh D, Truyers C, de Groot AM, Stoop J, Tete S, Van Damme W, Leroux-Roels I, Berghmans PJ. Interim results of a phase 1-2a trial of Ad26. COV2. S Covid-19 vaccine. New England Journal of Medicine. 2021 May 13;384(19):1824-35.

12. Bleier BS, Ramanathan Jr M, Lane AP. COVID-19 vaccines may not prevent nasal SARS-CoV-2 infection and asymptomatic transmission. Otolaryngology-Head and Neck Surgery. 2021 Feb;164(2):305-7.

13. Butt AA, Khan T, Yan P, Shaikh OS, Omer SB, Mayr F. Rate and risk factors for breakthrough SARS-CoV-2 infection after vaccination. J Infect 2021:83:237-79.

14. Tyagi K, Ghosh A, Nair D, Dutta K, Bhandari PS, Ansari IA, Misra A. Breakthrough COVID19 infections after vaccinations in healthcare and other workers in a chronic care medical facility in New Delhi, India.
Diabetes \& Metabolic Syndrome: Clinical Research \& Reviews. 2021 May 1; 15(3):1007-8.

15. Amit, S.; Regev-Yochay, G.; Afek, A.; Kreiss, Y.; Leshem, E. Early rate reductions of SARS-CoV-2 infection and COVID-19 in BNT162b2 vaccine recipients. Lancet 2021, 397, 875-877.

16. Hatmal MM, Al-Hatamleh MA, Olaimat AN, Hatmal M, Alhaj-Qasem DM, Olaimat TM, Mohamud R. Side Effects and Perceptions Following COVID-19 Vaccination in Jordan: A Randomized, Cross-Sectional Study Implementing Machine Learning for Predicting Severity of Side Effects. Vaccines. 2021 Jun;9(6):556

17. Zaqout A, Daghfal J, Alaqad I, Hussein SA, Aldushain A, Almaslamani MA, Abukhattab M, Omrani AS. The initial impact of a national BNT162b2 mRNA COVID-19 vaccine rollout. International Journal of Infectious Diseases. 2021 Jul 1;108:116-8

18. Ni L, Ye F, Cheng M-L, Feng Y, Deng Y-Q, Zhao H, et al. Detection of SARSCoV-2-specific humoral and cellular immunity in COVID-19 convalescent individuals. Immunity 2020;52

19. Hodgson SH, Mansatta K, Mallett G, Harris V, Emary KRW, Pollard AJ. What defines an efficacious COVID-19 vaccine? A review of the challenges assessing the clinical efficacy of vaccines against SARS-CoV-2. Lancet Infect Dis 2021;21:e26-e35.

20. Allam AA, Sayed AA. Active Covid-19 infection and transmission after the first dose of the BNT162b2 mRNA vaccination in Saudi Arabia: A case report. Journal of Infection and Public Health. 2021 Aug 1;14(8):1123-5.

21. Butt AA, Nafady-Hego H, Chemaitelly H, Abou-Samra AB, Al Khal A, Coyle PV, Al Kanaani Z, Kaleeckal AH, Latif AN, Al Masalmani Y, Bertollini R. Outcomes Among Patients with Breakthrough SARS-CoV-2 Infection After Vaccination: Breakthrough SARS-CoV-2 infection .International Journal of Infectious Diseases 110 (2021) 353-358.

22. Butt AA, Yan P, Shaikh OS, Mayr FB. Outcomes among patients with breakthrough SARS-CoV-2 infection after vaccination in a high-risk national population. E Clinical Medicine. 2021, 1;40:101117. 